

\title{
Migración Internacional Reciente de Honduras
}

\author{
Manuel Antonio Flores Fonseca*
}

RESUMEN. Este estudio es un recuento de la migración internacional reciente del país, principalmente de las últimas décadas, cuando se han producido transformaciones que hacen más visible la emigración, un acrecentamiento de los flujos, diversificación de destinos, cambios en los perfiles, que tienen su origen en diversas causas de origen económico, social, político y ambiental. Para ello, se utilizó como fuentes de datos demográficos, los censos de población, las encuestas de hogares o de migración, los registros administrativos de los países de origen y de destino. La migración se ha convertido en una estrategia de vida en la búsqueda de mejoras en las condiciones de vida, a través de un empleo, también se realiza para huir de la inseguridad ciudadana o simplemente buscar otros lugares para lograr movilidad social.

Palabras clave: Migración internacional, demografía.

ABSTRACT. This study is an account of recent international migration in the country, especially in recent decades, when changes have occurred that make it more visible migration, an increase of flows, diversification of destinations, profile changes, which have their origin in various causes of an economic, social, political and environmental. We use demographic data of population censuses, household surveys or migration, administrative records of the countries of origin and destination. Migration has become a livelihood strategy in the search for improvements in living conditions, through employment, fleeing insecurity or just find other hearths to achieve social mobility.

Keywords: International migration, demography.

\section{Introducción}

El objetivo de este estudio es presentar el panorama de la migración internacional reciente del país en las últimas décadas, partiendo de fuentes bibliográficas y de fuentes de datos demográficos en el país de origen y en los de destino, para detallar las transformaciones que han llevado a hacer más visible la emigración, el incremento de los volúmenes, cambios en los perfiles de los migrantes, y una mayor diversificación de destinos.

Aunque la migración siempre ha estado palpable en la sociedad hondureña, más que todo por su carácter inmigratorio, ya sea voluntario o por desplazamientos recibidos debido a conflictos bélicos, cierto es que en las dos últimas décadas se ha visibilizado en la población por la tendencia emigratoria, principalmente hacia los países de destino de mayor desarrollo.

En la migración internacional reciente de los hondureños prima la tendencia emigratoria, que presenta mayores dificultades metodológicas de medición, ya que tiene que tomar mano de censos, encuestas y registros de los países de destino y también de los mismos en el país de origen, donde muchas veces el acceso, oportunidad y calidad de la información no es tan óptima. Las estimaciones recientes y conservadoras de emigrantes muestran una ancha franja de diferencias, aunque su volumen es relevante para el país, tiene una tendencia creciente a través del tiempo, con características de juventud, altamente productivos, medianamente formados y cuyo destino emigratorio mayoritariamente continua siendo los Estados Unidos de América, pero se ha estado diversificando hacia otros destinos, incluso extra-regionales de Europa.

\section{Antecedentes}

La migración siempre ha estado presente a través de la historia hondureña, ya desde el descubrimiento, 
conquista y colonia son evidentes las políticas, leyes y acciones para promover y/o restringir los movimientos de poblaciones hacia el país, entre ellos los traslados de mano de obra negra del Caribe para el trabajo minero, los intentos de colonización blanca en la zona norte y la prohibición de asentamiento y comercio para algunos extranjeros, sin embargo, en la parte nororiental del litoral atlántico fue tierra de dominio de nacionales de Inglaterra y Francia donde se fundió un crisol de razas, blanca, negra, mulatos y mestizos. A finales de la colonia sobresale la llegada de negros de San Vicente que a través del tiempo se convierten en el grupo de afrodescendientes "garífuna" de tanta relevancia en el país.

Consecuentemente la época republicana a partir de 1821 y principalmente en la época de la reforma liberal de finales de ese siglo hasta la finalización del enclave minero y bananero en los años de 1930, las acciones gubernamentales propugnaron por leyes inmigratorias de atracción de poblaciones de preferencia estadounidenses y europeos, aunque no impedían la llegada de otras nacionalidades. Debido a que inmigrantes árabes fueron controlando el comercio se promulgan leyes migratorias discriminatorias para ciertas nacionalidades, como árabes, chinos y negros. Los judíos llegan en mayor medida a partir de los años treinta a Honduras (Amaya, 2000). Los inmigrantes entre las década de los treinta y cuarenta alcanzan las mayores proporciones con respecto a la población total, en los censos de 1926 y 1930 los extranjeros representan el $4.95 \%$ y en los censos de población de 1935 y 1940 los porcentajes son cercanos al 4\%, aunque en su mayoría los centroamericanos, estadounidenses y europeos representan porcentajes importantes del total de inmigrantes.

El declive del enclave bananero de los años treinta por la caída de las exportaciones causada por la recesión mundial y por enfermedades del banano empeoró la situación económica. Al mismo tiempo, empieza incipientemente los primeros avisos de tendencia emigratoria partiendo de la búsqueda de oportunidades primeramente de la población garífuna y mestiza conectada en la economía de enclave, principalmente a los puertos de la costa este de los Estados Unidos de América, para luego incluso llegar a alcanzar el primer lugar de la región como población emigrante en los años setenta, simultáneamente el país se fue transformando en atractivo para inmigrar en los años sesenta para la población salvadoreña en la búsqueda de tierras y trabajo y en los años setenta y ochenta por los conflictos armados centroamericanos que llevaron a nacionales de Guatemala, El Salvador y Nicaragua, a buscar refugio en el país.

Actualmente la tendencia migratoria claramente es la emigración, que se acelera en los años noventa con la implementación de las políticas económicas de corte neoliberal, que afectan más algunos sectores económicos, como el agrícola y generan una masa de jóvenes sin empleo o en calidad de cuentapropistas que son muy proclives a la búsqueda de oportunidades laborales en las regiones más desarrolladas del país y en gran medida en el exterior, principalmente en los Estados Unidos de América. Aparejado a esto se suma el desastre natural "Mitch" que hace visible la emigración como estrategia de vida, cuando los hondureños adquieren importancia numérica como migrantes por los flujos crecientes en la búsqueda del sueño americano.

En la última década, otros fenómenos sociales, económicos y políticos proliferan, tales como el creciente aparecimiento de maras y pandillas, organizaciones delictivas ligadas al narcotráfico, los niveles elevados de violencia y criminalidad ${ }^{1}$, el golpe de Estado del $2009^{2}$ y sus consecuencias, entre ellas la económica, a la que se agregó los efectos de la crisis económica mundial que ha llevado a deprimir la economía nacional en un

1. La violencia y la criminalidad ha aumentado en los últimos años en Honduras, los homicidios han tenido un comportamiento creciente en los últimos ocho años en un 229.7\%, en el 2004 ocurrieron un total de 2,155 muertes homicidas y para el 2011 esta cifra aumentó a 7,104 muertes acumulando en este período un total de 33,933 muertes. La tasa de homicidios pasó de 30.7 a 86.5 homicidios por 100.000 habitantes entre los años 2004 al 2011 , según el Observatorio Nacional de Violencia del IUDPAS-UNAH.

2. Un golpe de Estado el 28 de junio del 2009 derrocó al Presidente Constitucional de la República José Manuel Zelaya Rosales. 
marco de influencia neoliberal del gobierno actual ${ }^{3}$ que está incidiendo en un ambiente de poca generación de empleo, poca inversión y desde luego de aumento de la pobreza de la población ${ }^{4}$, que inciden en la búsqueda de oportunidades en otras tierras por medio de la migración.

\section{Marco de Referencia}

La migración es uno de los componentes principales de la dinámica demográfica, que fue altamente relegada en el pasado por los estudiosos de la población, tanto por las dificultades de medición y construcciones teóricas. Cierto es que a través del tiempo son visibles una serie de teorías explicativas de la migración desde diferentes disciplinas, entre ellas la Neoclásica, la nueva economía de las migraciones laborales, de los mercados duales, del sistema mundial, de las redes migratorias, del análisis de sistemas aplicados a las migraciones y la teoría de la causación acumulativa (Arango, 2003).

En el mundo la migración como variable demográfica aunque no ha decidido por sí misma el crecimiento demográfico, si contribuye en él y en los cambios en la estructura por edad y sexo de las poblaciones, tanto en los lugares de origen como en los de destino. Aunque siempre ha estado presente a través de la historia, son evidentes los desplazamientos en todo el mundo, motivados por diferentes causas, entre ellas las económicas por la búsqueda de mejores condiciones de vida, las de protección de la vida, por efecto de conflictos o desastres naturales, cierto es que en los últimos treinta años ha tomado relevancia en la opinión mundial como fenómeno que rebasa los aspectos demográficos.

La migración continua vigente con el incremento y diversificación de las demandas de información del tema y de las fuentes de datos tradicionales, como son los censos de población, las encuestas y los registros administrativos, ya que no completan ese conocimiento exhaustivo y es necesario ahondar en aspectos como los métodos de relevamientos de la información, estimaciones, cumplimiento con las disposiciones metodológicas generales de producción de datos y la explotación de más información. En la región, se han diversificado los requerimientos de estimación, se ampliaron la variedad de fuentes y la accesibilidad de datos, pero no ha cambiado la naturaleza de los procedimientos de estimación. Las principales fuentes de datos para la medición internacional en América Latina han sido los censos de población, mientras que los registros de entradas y salidas, aunque existieron con anterioridad, su uso ha sido escaso para fines estadísticos. Las encuestas de hogares que datan desde los años setenta con énfasis en el estudio de la fuerza de trabajo restringió su utilidad para fines de estudios migratorios (Calvelo, 2011).

Actualmente se estima en el mundo cerca de mil millones de migrantes (uno de cada siete de la población mundial), de los cuales 740 millones son migrantes internos y unos 214 millones son migrantes internacionales. Aunque las tendencias migratorias estaban marcadas en la década por fenómenos como la crisis económica mundial, el cambio climático y desplazamientos forzados, subyace cierta estabilidad general migratoria, pero se agregan eventos recientes como las llamadas transiciones políticas en Oriente Medio y África Septentrional, el aumento de los desastres naturales y sus desplazados, y la continuidad del número de refugiados y solicitantes de asilo (OIM, 2011). A estas tendencias se suma el endurecimiento de políticas inmigratorias de algunos países, incluso algunos estados de esos países, que atraen grandes flujos de migrantes que los hacen vivir penalidades, exclusión, irrespeto de los derechos humanos que los presiona a regresar a sus países de origen.

3. El gobierno de Porfirio Lobo (2010-2014) ha implementado una serie de medidas económicas como elevados aumentos de las tarifas de servicios públicos, mayor endeudamiento externo e interno, contraído los salarios, acelerado la depreciación de la moneda, nuevas tasas e impuestos, concesionado y privatizado en co-alianza con las empresas privadas varias actividades gubernamentales.

4. Según CEPAL en el 2010 en Honduras se incrementó significativamente sus tasas de pobreza e indigencia, de 1.7 y 1.0 puntos respectivamente. 
La región latinoamericana producto de las corrientes inmigratorias internacionales que había recibido a través del tiempo de otros continentes (desde la colonia hasta la mitad del siglo XX), había hecho que las fuentes de datos demográficas enfatizaran en la recolección de información de la inmigración, haciendo un vacío de instrumentos metodológicos para la medición de la emigración, aparte de que está adquirió más relevancia cuando aparecen o se expanden los destinos extra-regionales (Estados Unidos de América, Canadá, España y otros países).

Hay varias estimaciones de migrantes internacionales hondureños, en el caso de la inmigración no debería ser una complejidad actualmente, ya que existen registros de migración, aunque son de difícil acceso público, sin embargo, los censos de población han recogido información sobre el país de nacimiento y el país donde residía cinco años antes del censo, en el caso del censo de población de Honduras del 2001 los nacidos en el exterior ascienden a 27,976 personas. No pasa lo mismo con las estimaciones de emigrantes, que son más complejas, las estimaciones van desde utilizar los censos y encuestas de los países de origen y de destino y la utilización de procedimientos demográficos indirectos. Las estimaciones conservadoras más recientes muestran una ancha franja de diferencias, el Banco Mundial 2010 estima 569,700 emigrantes. La EPHPM 2010 estima 238,669, $\mathrm{ACS}^{4}$ 2009, registró 625,000 de origen hondureño en EUA, el censo de población de EUA 2010 estima 633,401 de origen hondureño, censos de población de ronda 2010 se calcula 677,950 nacidos y de origen hondureño (Flores, 2011). Los datos de ACS1 2010 relacionados con población de origen hondureño ascienden a 730,954 personas, sin embargo, un número más próximo de los emigrantes en las ACS sería tomar a los nacidos en Honduras que haría que dicha estimación sea de 529,312 personas.

\section{Metodología y Fuentes de Datos}

Este trabajo de tipo descriptivo presenta el panorama reciente de la migración internacional hondureña, tanto en el caso de la inmigración como de la emigración, utilizando fuentes bibliográficas y fuentes de datos demográficos nacionales e internacionales de datos generados especialmente para la elaboración de este estudio específico.

Las principales fuentes de datos utilizadas son los censos y encuestas nacionales (país de origen) donde se incluyen preguntas y en algunos casos módulos especiales sobre migración (interna e internacional), de ellos se rescata principalmente información de los inmigrantes en el país (que es más utilizada en las fuentes de datos demográficos), como también de aproximaciones de los emigrantes, de sus flujos, características, experiencias migratorias, partiendo del rescate de información que suministran los miembros de sus hogares de origen. Las limitaciones de las preguntas de emigrantes en los censos giran en torno a la definición adecuada del miembro del hogar y que se pueda identificar a los ex miembros del hogar, es decir, vivían, residían o pertenecían al hogar, por otro lado hay factores de recordación en el número acumulado de emigrantes a lo largo del tiempo, siendo más efectivo cuando el período de referencia de emigración es reciente. Los aspectos positivos de información censal de emigrantes son la universalidad y desagregación geográfica de la información, tendencias recientes de la emigración (características al momento de salir, flujos, países de destino, residencia actual), conocer características de los hogares con emigrantes (Maguid, 2009).

4. American Community Survey

5. Los censos de población hondureños en su mayoría han sido de facto, a excepción de los de 1974, 1988 y 2001 que son de jure o de derecho. 
Once de los diecinueve censos de población hondureños han recogido preguntas sobre el país de nacimiento, en los últimos tres censos sobre país de residencia cinco años antes del censo y en los dos últimos el año de llegada al país (1887, 1926, 1930, 1935, 1940, 1945, 1950, 1961, 1974, 1988 y 20015), así como el único que introdujo una pregunta sobre emigración de hondureños en un periodo cercano a la ocurrencia de un fenómeno natural (CNPV 20016). En el caso de la Encuesta Demográfica Nacional de Honduras, EDENH I 1972 y la EDENH II 1983 habían incursionado en las estimaciones de migración internacional, tanto de inmigrantes, como de emigrantes, en el caso de la segunda donde se incluyeron preguntas especiales para estimarla indirectamente basada en la residencia de ciertos familiares cercanos (madres, hijos o hermanos). Recientemente, es preciso mencionar que las Encuestas Permanentes de Hogares y de Propósitos Múltiples de Honduras (EPHPM), han llevado a cabo módulos especiales de migración en las correspondientes a los años 2006 y 2010.

Por otro lado, se utilizó el inventario de censos de población, encuestas de población o migratorias y los registros especiales de los países de destino, esto principalmente para rescatar los datos que permitan estimaciones sólidas del volumen de la emigración, los flujos migratorios y desde luego la caracterización de los emigrantes en los destinos. En ese sentido, se rescatan los datos del Proyecto de Investigación de la Migración Internacional (IMILA), del Banco Mundial (BM), así como de las propias Oficinas o Institutos de Estadística de varios países que generan información de migración, especialmente país de nacimiento, año de llegada al país, ascendencia, origen, ciudadanía, etc., en los censos de población a través de cuestionarios generales o en los cuestionarios ampliados, como es el caso de los Estados Unidos de América, que hace la operación censal puntualmente de manera decenal. Otros censos de población explorados son el de México, Canadá, países limítrofes y otros de la región y extra-regionales. No hay que olvidar que aunque no coincidan todos en los años de realización, se enmarcan en operaciones censales de la ronda de los años terminados en cero.

Otra fuente utilizada es la Encuesta de la Comunidad Americana (ACS) de Estados Unidos de América que posee una gran riqueza de información de interés demográfica, condiciones sociales, económicas y de vivienda. La ACS utiliza el concepto de residencia actual (viven o se quedan por más de dos meses) en comparación de los datos censales que utilizan el de residencia habitual, además que utiliza muestras mensuales para producir datos anuales. Estas encuestas han evolucionado en el tiempo, no sólo en el tamaño de la muestra de viviendas y población, utilización de viviendas particulares y viviendas colectivas institucionales y no institucionales y representatividad geográfica, sino que en el caso del 2010 reemplaza al cuestionario ampliado del censo de población (Gaspar, 2009). Las ACS utilizadas en este trabajo fueron especialmente las correspondientes a 2000 y del 2010, aunque existen grandes diferencias entre ellas en los aspectos señalados anteriormente, presentan un panorama de cambio decenal de utilidad demográfica, además de que las ACS son la mejor fuente de datos para conocer las características de la población nativa y de la población extranjera residente en ese país, que es de nuestro interés por ser el mayoritario destino emigratorio hondureño.

Otros registros de nuestro interés para el estudio de la emigración hondureña son en el caso de España, el Padrón Municipal Continuo que recoge información de la población, entre ella de los inmigrantes existentes en ese país, que tiene la ventaja que sus datos son muy actualizados y tiene incentivos para inscribirse, ya que no pide un estatus legal, y se tiene acceso a la educación y a los servicios de salud ${ }^{7}$, la desventaja es que incentiva

6. En el Censo de Población y Vivienda del 2001 se diseñó la Sección E. Migración Internacional. Pregunta 1. Después del huracán Mitch (octubre 1998), ¿alguna persona de este hogar se ha ido a vivir a otro país? Si/No. Pregunta 2. De esas personas que se fueron después del Mitch, icuántas viven actualmente en: a) Estados Unidos; b) Canadá; c) México; d) Centroamérica, e) Otros países?

7. Actualmente la cobertura sanitaria gratuita a los inmigrantes en situación irregular será restringido en España, salvo en las urgencias o embarazo, a partir del 1 de septiembre del 2012, por las medidas de austeridad del nuevo gobierno de Mariano Rajoy que espera ahorrar 7,000 millones de euros con esta medida. 
a inscribirse a personas que no residen habitualmente en España.

\section{Resultados}

\subsection{Volumen de Migrantes}

\subsubsection{Inmigrantes}

En el pasado la migración internacional del país estuvo influenciada hacia la inmigración, no sólo por las políticas e incentivos hacia ellas promulgadas a través de la historia, sino incluso por las fuentes de datos demográficas como los censos de población, los registros y las encuestas que han estado diseñadas a rescatar información mayoritariamente de la inmigración, que de la emigración, tendencia que ha variado recientemente.

Los datos de inmigración internacional rescatados de los censos de población hondureños muestran que sus valores siempre han sido bajos, generalmente entre $30 \mathrm{a}$ 50 mil personas y su porcentaje con respecto a la población total nunca ha sobrepasado el $5 \%$, sus porcentajes más altos datan de la década de los años treinta con una tendencia al descenso a través del tiempo, con los datos del último censo de población del 2001 alcanza sólo la mitad de uno por ciento. La mayoría procede de los países limítrofes (El Salvador, Nicaragua y Guatemala) influenciada principalmente por las conflictividades regionales, incluso un número no despreciable de estadounidenses (Cuadro No.1). Los registros administrativos de residentes extranjeros en el país también corroboran los valores bajos de inmigrantes.

Datos recientes de la EPHPM del 2010 estima un total de 37,666 hogares con personas nacidas en el extranjero, que equivalen a un $2.2 \%$ del total nacional, asimismo estiman un total de 46,883 personas nacidas en el extranjero (INE, 2011). Este valor es más elevado que los datos censales y de registros administrativos, aunque es más reciente y recoge datos con representación muestral en algunos dominios (nacional, rural, urbano, Tegucigalpa, San Pedro Sula, ciudades medianas), que puede incidir en los resultados.

\subsubsection{Emigrantes}

En las fuentes de datos demográficos hondureños no es sino hasta en la Encuesta Demográfica Nacional

Cuadro No. 1. Honduras: Población Nacida en Otros Países Empadronados en Censos Hondureños, 1887-2001

\begin{tabular}{|c|c|c|c|c|c|c|c|c|c|c|}
\hline Nacidos en & 1887 & 1926 & 1930 & 1935 & 1940 & 1945 & 1950 & 1961 & 1988 & 2001 \\
\hline Guatemala & 2,060 & 8,358 & 7,885 & 5,694 & 8,823 & 7,613 & 6,081 & 4,497 & 3,050 & 3,274 \\
\hline El Salvador & 2,000 & 13,452 & 18,522 & 19,168 & 21,309 & 23,029 & 20,285 & 38,002 & 7,733 & 6,291 \\
\hline Nicaragua & 610 & 3,162 & 5,907 & 4,614 & 3,298 & 2,183 & 2,760 & 3,553 & 15,149 & 5,519 \\
\hline Panamá & & & 77 & 95 & 75 & 64 & 105 & 159 & 178 & 283 \\
\hline Costa Rica & 14 & 182 & 178 & 207 & 203 & 162 & 275 & 294 & 557 & 611 \\
\hline Belice & & & 684 & & & & & & 200 & 259 \\
\hline Canadá & & & & & & & 11 & 82 & 149 & 248 \\
\hline Estados Unidos & 185 & 2,160 & 1,313 & 1,508 & 1,045 & 1,014 & 849 & 1,433 & 2,526 & 4,843 \\
\hline México & 29 & 343 & 424 & 535 & 382 & 300 & 302 & 379 & 746 & 1,114 \\
\hline Caribe & & 177 & 1,111 & 288 & 161 & 118 & 227 & 306 & 294 & 761 \\
\hline Sudamérica & 16 & 22 & 118 & 148 & 218 & 97 & 170 & 393 & 1,578 & 1,991 \\
\hline Europa & 1,252 & 5,544 & 4,358 & 4,710 & 3,664 & 3,204 & 1,217 & 1,598 & 837 & 1,234 \\
\hline Asia & 1 & 1,287 & 1,063 & 1,249 & 1,208 & 1,176 & 382 & 479 & 441 & 1,054 \\
\hline África & & & 8 & & & & 39 & & & 25 \\
\hline Otros & & 27 & 632 & 280 & 1,026 & 71 & & 29 & 949 & 469 \\
\hline Total & 6,167 & 34,714 & 42,280 & 38,496 & 41,412 & 39,031 & 32,703 & 51,204 & 34,387 & 27,976 \\
\hline
\end{tabular}

Fuente: Elaboración propia en base a datos de los Censos de Población de Honduras, 1887-2001, DGECH e INE. 
de Honduras (EDENH II de 1983) que se encuentra una medición de la emigración internacional utilizando métodos indirectos, estimando un 3.4\% la población que reside en el exterior.

En el censo de población hondureño del 2001 por primera vez se incluye una pregunta sobre emigración internacional, relativa a hogares con emigrantes en un intervalo corto de tiempo, esta fuente estima que en el 3.34\% de los hogares hondureños alguna persona había salido del país en los tres años anteriores al censo, teniendo como referencia temporal el huracán Mitch, un fenómeno natural que quedó en la memoria colectiva de la población (Flores, 2010).

La EPHPM de mayo del 2010 incluye un módulo de migración que estima unos 168,786 hogares con población emigrante internacional, que representa un $9.7 \%$ del total nacional. En el caso de la población emigrante hondureña estima 238,669 personas. Cuatro años antes, la EPHPM de mayo de 1996 había estimado datos más elevados, un total de 179,051 hogares que es un $11.3 \%$ del total y 246,620 emigrantes, que podría indicar una desaceleración de la emigración. Hay que tomar en cuenta que los censos y las encuestas realizadas en los países de origen subestiman la emigración por algunas razones, entre ellas, que la información es suministrada en el hogar de origen del emigrante por sus familiares o informantes, que hace que se necesite un buen manejo metodológico en su recopilación.

Con datos de IMILA e Institutos de Estadística de varios países, se estimó que en la década de los años setenta habría un número mínimo de 42,737 hondureños residiendo en el exterior, en la ronda de los censos de los ochenta se estimaría unos 50,930 emigrantes. En los años noventa 142,274 hondureños, en la ronda de censos del dos mil 322,696 y en los censos de la ronda del $2010^{8}$ unos 645,532 residiendo en el extranjero como dato mínimo (Cuadro No.2).

\subsection{Principales Destinos Emigratorios.}

Como la emigración es la tendencia predominante en las últimas décadas. Los principales destinos emigratorios son los Estados Unidos de América, España, México, los países de Centroamérica y el resto los demás países.

\section{Cuadro No. 2. Honduras: Población Nacional Empadronada en Censos de Po- blación en Países de Destino, 1970-2010}

\begin{tabular}{|c|c|c|c|c|c|}
\hline País de empadronamiento & 1970 & 1980 & 1990 & 2000 & 2010 \\
\hline Argentina & 88 & & 138 & 142 & \\
\hline Belice & & 1,576 & 2,337 & & \\
\hline Bolivia & 204 & & 189 & 195 & \\
\hline Brasil & 83 & 207 & 300 & 136 & \\
\hline Colombia & & & 182 & & \\
\hline Costa Rica & 996 & 1,574 & & 2,946 & \\
\hline \multicolumn{6}{|l|}{ Cuba } \\
\hline Chile & 122 & 179 & 220 & 244 & \\
\hline Ecuador & & 104 & 117 & 195 & \\
\hline El Salvador & 14,290 & & 8,666 & 10,387 & \\
\hline Guatemala & 6,231 & 5,326 & 4,634 & 5,491 & \\
\hline Haití & 7 & & & & \\
\hline México & 941 & 1,500 & 1,997 & 3,570 & 10,991 \\
\hline Nicaragua & 6,919 & & 9,473 & 10,745 & \\
\hline Panamá & & 464 & 623 & 823 & 1,140 \\
\hline Paraguay & & 27 & 35 & & \\
\hline Perú & & & 111 & & \\
\hline Rep. Dominicana & 32 & & & 143 & \\
\hline Uruguay & & & 17 & & \\
\hline Venezuela & 165 & 344 & 429 & 252 & \\
\hline Canadá & & 475 & 3,935 & 4,575 & \\
\hline Estados Unidos de América & 27,978 & 39,154 & 108,923 & 282,852 & 633,401 \\
\hline Total & 58,056 & 50,930 & 142,326 & 322,696 & 645,532 \\
\hline
\end{tabular}

Fuente: Elaboración propia en base a datos de IMILA e Institutos de Estadística de los países. 
El primer destino emigratorio por excelencia sigue siendo los Estados Unidos de América, esto lo evidencian todas las fuentes de datos demográficos ${ }^{9}$. En el caso de los censos estadounidenses las personas de origen hondureño han aumentado en el tiempo, aunque su tasa de crecimiento anual tiene una tendencia hacia el descenso en los últimos veinte años (Cuadro No. 3).

La ACS de varios años recientes y el último censo de población de los Estados Unidos, muestra que la población de origen hondureño y la que nació en Honduras y que reside allí, sigue en aumento en su presencia en ese país del norte (Cuadro No.4).

España como segundo destino emigratorio hondureño ha mostrado un crecimiento elevado en la última década, pasando de 2,236 en el año dos mil a 32,418 en el año 2011, siendo los últimos cinco años el periodo de mayor aumento (Cuadro No.5). Esta migración ha llevado

Cuadro No. 3. Estados Unidos de América: Población de Origen Hondureño en Censos de Población, 1970-2010

\begin{tabular}{|c|c|c|c|c|}
\hline Año & Población & $\begin{array}{c}\text { Incremento } \\
\text { intercensal }\end{array}$ & $\begin{array}{c}\% \text { de } \\
\text { incremento } \\
\text { intercensal }\end{array}$ & $\begin{array}{c}\text { Tasa anual } \\
\text { de creci- } \\
\text { miento }\end{array}$ \\
\hline 1970 & 27,978 & & & \\
\hline 1980 & 39,154 & 11,176 & 39.95 & 3.3 \\
\hline 1990 & 108,923 & 69,769 & 178.19 & 9.4 \\
\hline 2000 & 282,850 & 173,927 & 159.68 & 8.9 \\
\hline 2010 & 633,401 & 350,551 & 123.94 & 7.7 \\
\hline
\end{tabular}

Fuente: IMILA, CELADE y Census Bureau de EUA, 2010

Cuadro No. 4. Estados Unidos de América: Población de Origen y de Nacimiento en Honduras, 2005-2010

\begin{tabular}{|c|c|c|c|}
\hline Año & $\begin{array}{c}\text { Censo de } \\
\text { Población }\end{array}$ & $\begin{array}{c}\text { ACS origen } \\
\text { hondureño }\end{array}$ & $\begin{array}{c}\text { ACS nació en } \\
\text { Honduras }\end{array}$ \\
\hline 2005 & & 466,843 & 393,349 \\
\hline 2006 & & 486,026 & 406,097 \\
\hline 2007 & & 527,154 & 429,931 \\
\hline 2008 & & 607,970 & 466,450 \\
\hline 2009 & & 624,533 & 468,614 \\
\hline 2010 & 633,401 & 730,954 & 529,312 \\
\hline
\end{tabular}

Fuente: Elaboración propia de datos de Bureau Census de EUA y generados de bases de ACS, 2005-2010. a convertir a los hondureños en la primer colonia de centroamericanos en ese país de Europa, duplicando su peso del total, de $20.5 \%$ en el 2000 ha llegado a $43.9 \%$ en el $2010^{10}$.

Otro destino importante de los emigrantes hondureños es México, que también muestra aumentos en los últimos años, no sólo en el volumen, sino en el crecimiento intercensal (Cuadro No.6). No hay que olvidar que esta cifra incluye solamente los empadronados, ya que México como país en ruta hacia los Estados Unidos alberga un número importante de hondureños en tránsito.

\section{Cuadro No.5. España: Población Nacida en Honduras por Sexo Según Año, 1998-2011}

\begin{tabular}{|c|c|c|c|}
\hline Año & Hombres & Mujeres & Total \\
\hline 1998 & 520 & 1,151 & 1,671 \\
\hline 1999 & 570 & 1,254 & 1,824 \\
\hline 2000 & 694 & 1,542 & 2,236 \\
\hline 2001 & 991 & 2,022 & 3,013 \\
\hline 2002 & 1,355 & 2,593 & 3,948 \\
\hline 2003 & 1,848 & 3,395 & 5,243 \\
\hline 2004 & 2,290 & 4,071 & 6,361 \\
\hline 2005 & 3,001 & 5,321 & 8,322 \\
\hline 2006 & 3,840 & 6,812 & 10,652 \\
\hline 2007 & 5,498 & 10,396 & 15,894 \\
\hline 2008 & 7,844 & 15,829 & 23,673 \\
\hline 2009 & 8,648 & 18,186 & 26,834 \\
\hline 2010 & 8,820 & 20,031 & 28,851 \\
\hline 2011 & 9,392 & 23,026 & 32,418 \\
\hline
\end{tabular}

Fuente: Instituto de Estadística INE, España.

Cuadro No. 6. México: Población Residente Nacida en Honduras, 1970-2010

\begin{tabular}{|c|c|c|c|c|}
\hline Año & Población & $\begin{array}{c}\text { Incremento } \\
\text { intercensal }\end{array}$ & $\begin{array}{c}\text { Te de } \\
\text { incremento } \\
\text { intercensal }\end{array}$ & $\begin{array}{c}\text { Tasa anual } \\
\text { de creci- } \\
\text { miento }\end{array}$ \\
\hline 1970 & 942 & & 59.24 & 4.7 \\
\hline 1980 & 1,500 & 558 & 33.13 & 2.9 \\
\hline 1990 & 1,997 & 497 & 86.38 & 6.2 \\
\hline 2000 & 3,722 & 1,725 & 195.30 & 10.8 \\
\hline 2010 & 10,991 & 7,269 & & \\
\hline
\end{tabular}

Fuente: Elaboración propia basada en las estadísticas históricas de México, INEGI.

9. Los módulos de migración de la EPHPM 2006, 91.4\% de la población migrante reside en EUA y en EPHPM 2010 es $88 \%$ hacia EUA.

10. Los centroamericanos (Guatemala, Honduras, El Salvador, Nicaragua, Costa Rica y Panamá) en España eran en el 2000 unos 10,883 y en el 2010 alcanzan los 65,694 personas. 
En ese sentido, la Encuesta de Migración de la Frontera Sur (ENIF SUR) encuentra durante el segundo semestre del 2008 que 23,305 migrantes centroamericanos que declararon tener intención de llegar a los Estados Unidos de América, fueron devueltos por las autoridades mexicanas; de los cuales el 39\% era de Honduras $(9,015)$, mayoritariamente hombres, en edades jóvenes, con educación entre 6 y 9 años (71.1\%), el 40.2\% detenido dos o más veces en México y el $84.4 \%$ reintentará cruzar nuevamente para ingresar a los Estados Unidos. Al mismo tiempo la ENIF Sur para tener un panorama completo de la migración en tránsito por México incluye los devueltos por las autoridades norteamericanas en el periodo señalado, que asciende a 40,744 centroamericanos de los cuales 13,218 hondureños (INM y otros, 2011).

Cuadro No. 7. Canadá: Población de Origen Hondureño
en los Censos de Población, 1981-2006
\begin{tabular}{|c|c|c|c|c|}
\hline Año & Población & $\begin{array}{c}\text { Incremento } \\
\text { intercensal }\end{array}$ & $\begin{array}{c}\% \text { de incre- } \\
\text { mento inter- } \\
\text { censal }\end{array}$ & $\begin{array}{c}\text { Tasa anual } \\
\text { de creci- } \\
\text { miento }\end{array}$ \\
\hline 1981 & 475 & & & \\
\hline 1986 & 865 & 390 & 82.11 & 11.6 \\
\hline 1996 & 3,935 & 3,070 & 354.91 & 12.8 \\
\hline 2001 & 4,575 & 640 & 16.26 & 3.0 \\
\hline 2006 & 5,165 & 590 & 12.90 & 2.4 \\
\hline
\end{tabular}

Fuente: Elaboración propia en base a datos censales de Canadá.

Canadá como destino emigratorio de hondureños es visible, ya que a través del tiempo se ha incrementado (Cuadro No.7), aun cuando las políticas migratorias canadienses son cada vez más restrictivas y se convierten en un impedimento para optar a residir ese país del norte.

\subsection{Características de los Emigrantes.}

La EPHPM de mayo 2010 en su módulo de migración caracteriza a la población emigrante, partiendo de la información que suministran sus familiares o informantes del hogar de donde sale. Los emigrantes hondureños en su mayoría son hombres (2/3 del total), se concentran más en el área rural (54.6\%), la estructura por edad joven, principalmente entre 20 a 34 años, mayoritariamente era hijo en su relación de parentesco con el jefe al momento de salir del país (65\%), el 79\% tenía algún nivel educativo al momento de salir del país, aunque mayoritariamente disponía de solamente algún grado de la educación básica, el 29\% residía de manera legal en el país donde se encuentra (incluyendo visas de turista, estudiantes, trabajar, residentes, nacionalizados y amparados). Los principales destinos de los emigrantes son EUA un 88\%, España 5.7\%, México 2.5\%, Centroamérica $1.9 \%$ y otros países $1.9 \%$.

\subsubsection{En los Estados Unidos de América.}

Con datos de la ACS 2009 se hizo una caracterización de la población de origen hondureño en los Estados Unidos de América, detallando unos 625 mil personas que los ubica en el octavo lugar de la población de origen hispano, siendo mayoritariamente jóvenes, con una edad mediana de 28 años, con edades ubicadas en dos grupos mayoritarios, los menores de cinco años y los ubicados en edades activas entre los veinte a cincuenta años, un índice de masculinidad de 118. De la población de origen hondureño 7 de cada 10 nació en el extranjero (más cercanamente del valor de emigrantes), había llegado mayoritariamente a ese país en 1990 ó después y solamente el 20\% era ciudadano estadounidense. Sólo el 40\% habla inglés con fluidez y más del 70\% vive en el sur del país, principalmente en los estados de la Florida y Texas, en California el 13\% y Nueva York el 9\%. En sus características educativas se muestra el bajo nivel de educación, aún comparado con los promedios de población hispana, que hace que la mitad de los mayores de 25 años no ha obtenido un diploma de educación secundaria. Los ingresos anuales alcanzan solamente los 17,100 dólares (bajo para el promedio hispano), el 27\% vive en la pobreza, la mitad de ellos no tiene seguro de salud y las tasas de propiedad es de 32\% (PEW, 2011).

Una comparación de datos de la población de origen hondureño en las ACS 2000 y 2010 resultan evidentes algunos cambios en algunas variables demográficas, sociales y económicas. El volumen de la población se incrementó tres veces en la década. En el sexo es muy similar su comportamiento en el tiempo, alrededor del $52 \%$ son hombres, por grupos de edades el índice de masculinidad 
es superior a cien hasta los treinta y cuarenta años, en adelante hay una supremacía femenina hasta las edades superiores. Por otro lado, la pirámide de la población de origen hondureño está concentrada en las edades jóvenes de 20 a 40 años, sin embargo, hay un proceso de rejuvenecimiento de la población, mostrado en las poblaciones de la niñez (Gráfico No.1). En las relaciones de parentesco sobresale en la década el aumento del peso de los hijos y de los amigos y compañeros, en cambio hay reducciones en los jefes de hogar y los cónyuges.

En otras características sociodemográficas, el estado civil de la población de origen hondureño ha variado significativamente hacía la reducción relativa de los casados con cónyuge presente y con el aumento de los solteros nunca casados. En la raza mayoritariamente se declara como blanca o como otros; en términos de volumen hubo un cambio en la declarada raza blanca que se incrementó en la década cuatro veces y la categoría otros también se incrementó casí tres veces. La población declarada negra duplicó su número, siendo el grupo hondureño el de mayor experiencia migratoria internacional. Estos cambios a nivel relativo contrariamente produjeron un menor peso de la población no blanca en todas las categorías.

Aunque hay un incremento relativo en el número de originarios de Honduras que se declaran ciudadanos naturalizados, la inmensa mayoría se declara no ciudadano estadounidense. La categoría de ciudadanos naturalizados ha perdido peso levemente, pero los declarados no ciudadanos aumentan sus porcentajes del total.

De las personas de origen hondureño encuestadas en las Encuestas de la Comunidad Americana según el país de nacimiento en la década es visible su crecimiento absoluto en las tres categorías (nacido en EUA, otro país, Honduras), aunque los nacidos en los Estados Unidos de América incrementó 4.6 veces, casi el doble de los nacidos en Honduras. Sin embargo, en términos relativos sólo ocurre el aumento en los nacidos en EUA, las demás categorías descienden, principalmente en los nacidos en Honduras, de quienes es preciso señalar están más cercanos a la categoría de inmigrantes en el país del norte (Cuadro No. 8).

\section{Gráfico No. 1. EUA: Pirámide de Población de Origen Hondureño, 2000-2010}

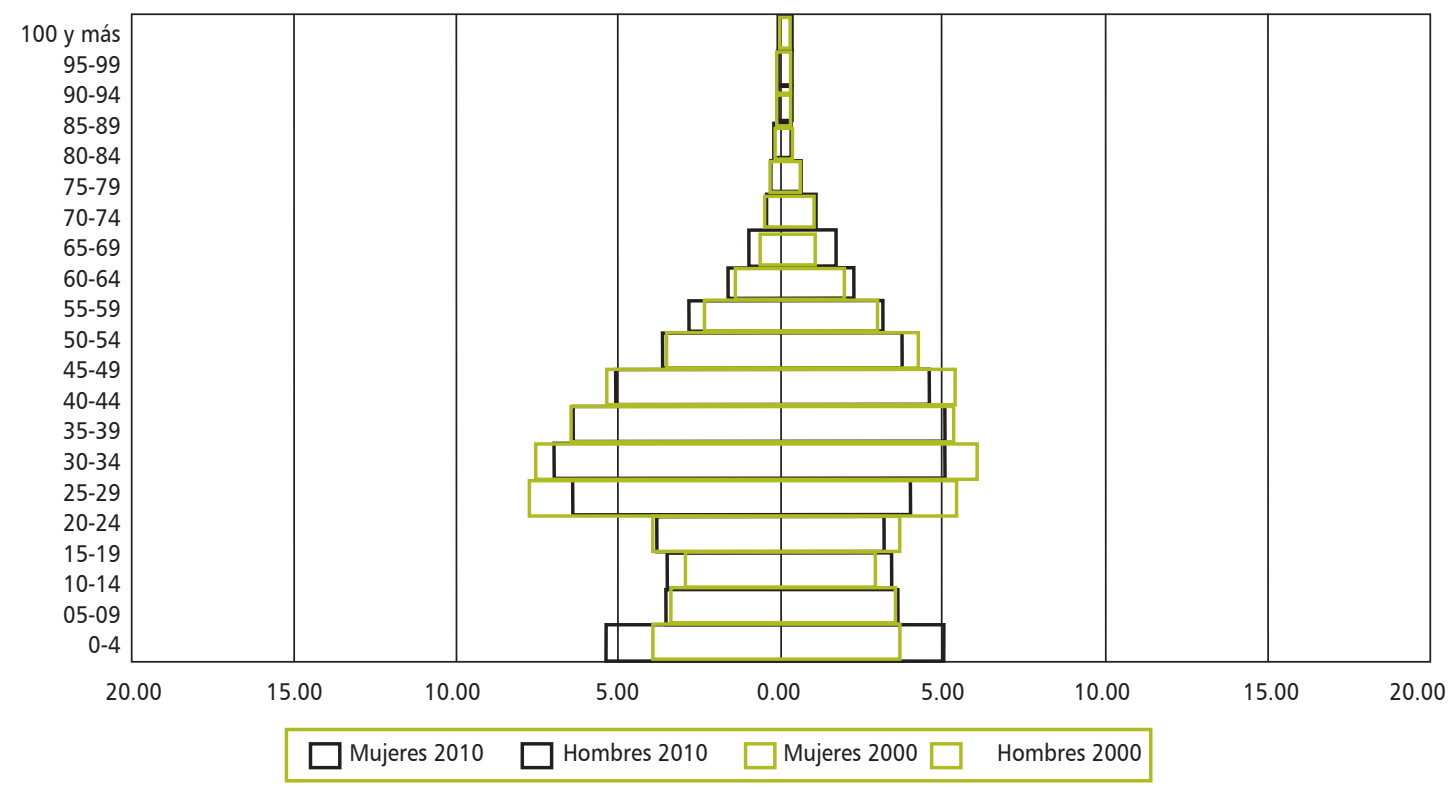

Fuente: ASC Encuesta de la Comunidad Americana, 2000 - 2010 
Cuadro No. 8. EUA: Población de Origen Hondureño Según País de Nacimiento, 2000-2010

\begin{tabular}{|l|c|c|c|c|}
\hline $\begin{array}{l}\text { País de } \\
\text { Nacimiento }\end{array}$ & $\begin{array}{c}2000 \\
\text { Abs. }\end{array}$ & $\begin{array}{c}2010 \\
\text { Abs. }\end{array}$ & $\begin{array}{c}2000 \\
\text { Rel. }\end{array}$ & $\begin{array}{c}2010 \\
\text { Rel. }\end{array}$ \\
\hline EUA & 51,145 & 236,060 & 21.6 & 32.3 \\
\hline Otro País & 8,883 & 17,630 & 3.7 & 2.4 \\
\hline Honduras & 177,103 & 477,264 & 74.7 & 65.3 \\
\hline Total & 237,131 & 730,954 & 100.0 & 100.0 \\
\hline
\end{tabular}

Fuente: Elaboración propia generada de base de datos ACS 2000 y 2010.

En los años de residencia en los Estados Unidos son visibles los cambios, principalmente en los que tienen cinco años o menos y en los que tienen más de veinte años de residir en el país de destino (Cuadro No.9).

Aunque las condiciones de bienestar de la población de origen hondureño no son las mejores si las comparamos con los promedios de la población de origen hispano, si indican mejoría con respecto al país de origen y también en el país de destino a través del tiempo, aunque no en la magnitud deseada como población migrante. Algunas características relevantes en la población de origen hondureño en los Estados Unidos de América permiten observar en una década cambios muy lentos, en el caso de la proficiencia del inglés y la asistencia escolar, en el caso del logro educativo se incrementó un 5\% la población con estudios arriba de la educación media en la década (35.2\% a 40.7\%). En el caso laboral ha aumentado la proporción de personas en la fuerza laboral que está empleado y que generalmente trabaja por salario, aunque ha aumentado la proporción de auto-empleados. En el estado de residencia hay una mayor dispersión en los estados en que viven, aunque los de preferencia residencial continúan

Cuadro No. 9. EUA: Población de Origen Hondureño Según Años en los EUA, 2000-2010

\begin{tabular}{|l|c|c|}
\hline Años en EUA & 2000 & 2010 \\
\hline $0-5$ años & 36.3 & 26.3 \\
\hline $6-10$ años & 23.0 & 23.6 \\
\hline $11-15$ años & 17.4 & 17.1 \\
\hline $16-20$ años & 10.3 & 12.4 \\
\hline $21+$ años & 13.0 & 20.6 \\
\hline Total & 100.0 & 100.0 \\
\hline
\end{tabular}

Fuente: Elaboración propia generada de base de datos ACS 2000 y 2010. siendo los de la costa este, el sur y en el extremo oeste (Cuadro No. 10).

Cuadro No. 10. ACS: Población de Origen Hondureño en EUA Según Algunas Características, 2000-2010

\begin{tabular}{|c|c|c|}
\hline Característica & 2000 & 2010 \\
\hline \multicolumn{3}{|l|}{ Habla inglés } \\
\hline No & 15.2 & 14.9 \\
\hline Si, solo inglés & 8.8 & 8.6 \\
\hline Si, muy bien & 30.5 & 33.5 \\
\hline $\mathrm{Si}$, bien & 20.2 & 19.1 \\
\hline Si, pero no bien & 25.4 & 24.0 \\
\hline \multicolumn{3}{|l|}{ Asistencia a escuela } \\
\hline No, esta en la escuela & 74.4 & 73.1 \\
\hline Si, esta en la escuela & 25.6 & 26.9 \\
\hline \multicolumn{3}{|l|}{ Logro educativo } \\
\hline Escuela no completa & 9.4 & 6.9 \\
\hline Nursery hasta 4 grado & 10.0 & 13.0 \\
\hline Grado 5 hasta 12 & 45.4 & 39.5 \\
\hline Graduado bachillerato & 16.9 & 19.7 \\
\hline Algún college menos de 1 año & 3.4 & 2.0 \\
\hline College 1 ó más años, pero sin grado & 6.6 & 9.3 \\
\hline Grado asociaciado sin especificar & 2.4 & 3.0 \\
\hline Licenciatura & 3.8 & 5.0 \\
\hline Maestría & 1.0 & 1.1 \\
\hline Grado profesional más alla de licenciatura & 0.8 & 0.4 \\
\hline Doctorado & 0.2 & 0.1 \\
\hline \multicolumn{3}{|l|}{ Estatus Empleo } \\
\hline Empleado & 61.0 & 66.3 \\
\hline Desempleado & 6.8 & 9.7 \\
\hline No, está en fuerza laboral & 32.2 & 24.0 \\
\hline \multicolumn{3}{|l|}{ Estatus fuerza laboral } \\
\hline No, esta en fuerza laboral & 32.2 & 24.0 \\
\hline Si, esta en fuerza laboral & 67.8 & 76.0 \\
\hline \multicolumn{3}{|l|}{ Clase de trabajo } \\
\hline Autoempleado & 6.9 & 11.7 \\
\hline Trabaja por salario & 93.1 & 88.3 \\
\hline \multicolumn{3}{|l|}{ Estado de Residencia (ICPSR) } \\
\hline Florida & 18.5 & 17.6 \\
\hline Texas & 11.2 & 14.1 \\
\hline New York & 16.3 & 11.9 \\
\hline California & 14.8 & 10.9 \\
\hline New Jersey & 6.9 & 5.4 \\
\hline North Carolina & 3.8 & 5.1 \\
\hline Virginia & 3.1 & 5.0 \\
\hline Lousiana & 4.6 & 4.8 \\
\hline Otros & 20.8 & 25.2 \\
\hline
\end{tabular}

Fuente: Elaboración propia generada de base de datos ACS 2000 y 2010. 


\subsubsection{En España}

España es un destino extra regional de la migración hondureña que ha adquirido una importancia muy grande en la última década. El padrón español al 1 de enero del 2011 registró un total de 32,418 nacidos en Honduras, de las cuales la mayoría son mujeres, 7 de cada 10. La población es mayoritariamente joven, ubicada entre los 20 a los 49 años, donde el grupo más representativo esta en las edades entre 25 a 34 años, principalmente mujeres (Gráfico No.2).

La estructura por edad y sexo en la Comunidad de Cataluña, una de las que absorbe el mayor número de nacidos en Honduras, muestra también concentraciones en las edades productivas, principalmente en las mujeres, que son el grupo mayoritario. La provincia de Barcelona y Girona reproducen en la población extranjera nacida en Honduras ese comportamiento en la estructura por edad y sexo.

En relación a las comunidades y provincias de residencia en España los nacidos en Honduras residen principalmente en las Comunidades de Cataluña, Madrid, Valencia y País Vasco. Específicamente en la Comunidad de Cataluña vive la mitad de los hondureños, principalmente en las Provincias de Barcelona (26.4\%) y Girona (21.7\%), cercanas a Francia, que dicho sea de paso se desconocen los motivos de esta ubicación geográfica y que es preciso investigar. En el caso de las otras comunidades de residencia, sobresalen las Provincias de Madrid, Valencia y Guipuzcoa (Cuadro No.11).

\section{Gráfico No. 2. España: Población Hondureña en el Padrón Municipal 1 de enero 2011}

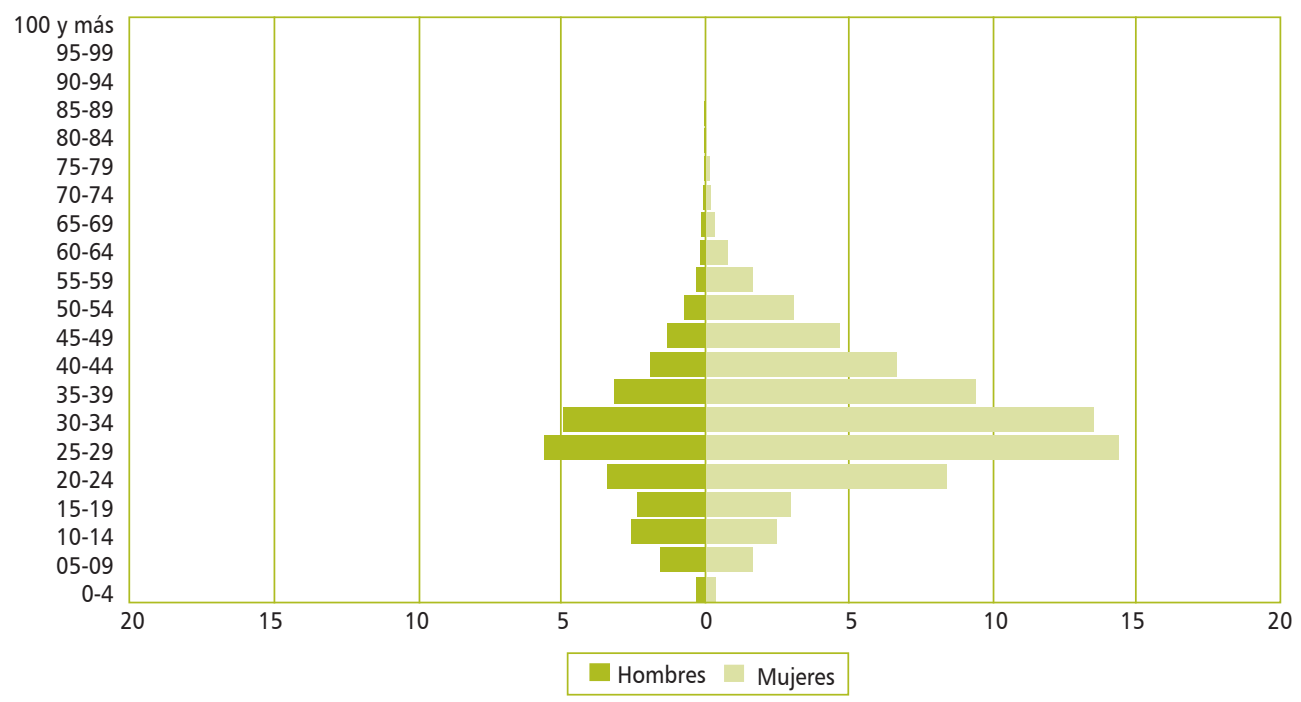

Fuente: elaboración propia, en base al padrón español INE, 2011 
Cuadro No. 11. España: Población Nacida en Honduras Según Comunidad Autónoma y Provincias, 2011

\begin{tabular}{|c|c|c|c|c|c|c|c|}
\hline $\begin{array}{l}\text { Comunidad Autónoma y } \\
\text { Provincia }\end{array}$ & Hombres & Mujeres & $\begin{array}{l}\text { Ambos } \\
\text { sexos }\end{array}$ & $\begin{array}{l}\text { Comunidad Autónoma y Pro- } \\
\text { vincia }\end{array}$ & Hombres & Mujeres & $\begin{array}{l}\text { Ambos } \\
\text { sexos }\end{array}$ \\
\hline ANDALUCÍA & 308 & 779 & 1,087 & Cuenca & 13 & 20 & 33 \\
\hline Almería & 35 & 71 & 106 & Guadalajara & 37 & 104 & 141 \\
\hline Cádiz & 48 & 115 & 163 & Toledo & 94 & 162 & 256 \\
\hline Córdoba & 25 & 101 & 126 & CATALUÑA & 4,818 & 11,330 & 16,148 \\
\hline Granada & 40 & 107 & 147 & Barcelona & 2,508 & 6,081 & 8,589 \\
\hline Huelva & 12 & 15 & 27 & Girona & 2,182 & 4,884 & 7,066 \\
\hline Jaén & 19 & 36 & 55 & Lleida & 36 & 89 & 125 \\
\hline Málaga & 61 & 154 & 215 & Tarragona & 92 & 276 & 368 \\
\hline Sevilla & 68 & 180 & 248 & COMUNITAT VALENCIANA & 618 & 1,661 & 2,279 \\
\hline ARAGÓN & 254 & 572 & 826 & Alicante/Alacant & 123 & 266 & 389 \\
\hline Huesca & 20 & 48 & 68 & Castellón/Castelló & 49 & 103 & 152 \\
\hline Teruel & 4 & 6 & 10 & Valencia/València & 446 & 1,292 & 1,738 \\
\hline Zaragoza & 230 & 518 & 748 & EXTREMADURA & 75 & 249 & 324 \\
\hline ASTURIAS, PRINCIPADO DE & 33 & 82 & 115 & Badajoz & 23 & 97 & 120 \\
\hline BALEARS, ILLES & 69 & 133 & 202 & Cáceres & 52 & 152 & 204 \\
\hline CANARIAS & 154 & 380 & 534 & GALICIA & 89 & 194 & 283 \\
\hline Palmas, Las & 122 & 318 & 440 & Coruña, $A$ & 36 & 70 & 106 \\
\hline Santa Cruz de Tenerife & 32 & 62 & 94 & Lugo & 18 & 41 & 59 \\
\hline CANTABRIA & 27 & 60 & 87 & Ourense & 12 & 29 & 41 \\
\hline CASTILLA Y LEÓN & 467 & 929 & 1,396 & Pontevedra & 23 & 54 & 77 \\
\hline Ávila & 7 & 19 & 26 & MADRID, COMUNIDAD DE & 1,638 & 4,613 & 6,251 \\
\hline Burgos & 120 & 246 & 366 & MURCIA, REGIÓN DE & 149 & 285 & 434 \\
\hline León & 22 & 26 & 48 & NAVARRA, COMUNIDAD FORAL DE & 54 & 164 & 218 \\
\hline Palencia & 7 & 13 & 20 & PAÍS VASCO & 447 & 1,271 & 1,718 \\
\hline Salamanca & 97 & 181 & 278 & Araba/Álava & 15 & 51 & 66 \\
\hline Segovia & 172 & 354 & 526 & Bizkaia & 125 & 318 & 443 \\
\hline Soria & 11 & 34 & 45 & Gipuzkoa & 307 & 902 & 1,209 \\
\hline Valladolid & 31 & 48 & 79 & RIOJA, LA & 17 & 34 & 51 \\
\hline Zamora & 0 & 8 & 8 & Ceuta & 1 & 0 & 1 \\
\hline CASTILLA - LA MANCHA & 199 & 373 & 572 & Melilla & 1 & 0 & 1 \\
\hline Albacete & 24 & 44 & 68 & TOTAL ESPAÑA & 9,418 & 23,109 & 32,527 \\
\hline Ciudad Real & 31 & 43 & 74 & & & & \\
\hline
\end{tabular}

Fuente: Elaboración propia generada del Padrón Municipal, INE, España.

\section{Conclusiones}

La emigración es la tendencia de relevancia en las últimas dos décadas en el comportamiento de la migración en Honduras, con una tendencia creciente, duplicándose cada década. Los principales destinos emigratorios son los Estados Unidos de América, España, México, los países de Centroamérica y el resto los demás países. El primero es el destino mayoritario por excelencia.

La población de origen hondureño en los Estados Unidos de América la ubica en el octavo lugar de la población de origen hispano, con un perfil demográfico mayoritariamente joven, con una edad mediana de 28 años y un índice de masculinidad de 118. Las condiciones de bienestar de la población de origen hondureño no son las mejores, si las comparamos con los promedios de la población de origen hispano, se observan cambios en algunas variables, aunque no en la magnitud deseada como población migrante.

España surge como destino extra-regional de importancia para la emigración hondureña, con un perfil emigrante mayoritariamente femenino y concentrado en pocas comunidades y provincias. 


\section{Bibliografía}

- Amaya Banegas, Jorge (2000). Los judíos en Honduras, Tegucigalpa, Guaymuras.

- Arango, Joaquín (2003). La Explicación Teórica de las Migraciones: Luz y Sombra, Migración y Desarrollo no 1 , octubre 2003.

- Calvelo, Laura (2011). Viejos y nuevos asuntos en las estimaciones de migración internacional en América Latina y el Caribe, Santiago, CELADE-CEPAL.

- Flores Fonseca, Manuel Antonio (2010). "La Medición Censal de la Migración en Honduras", Notas de Población no 88, Santiago, CEPAL.

- Flores Fonseca, Manuel Antonio (2011). Tendencias Migratorias de la Migración Internacional de Honduras, San Francisco de las casas, Chiapas, presentado en 3er. Coloquio de Migración Internacional, IIESUNAH.

- Gaspar Olvera, Selene (2009). La migración internacional a través de los censos y encuestas de los Estados Unidos, SIMDE-UAZ, 2009.

- INE (2011). Comportamiento de la Migración y Re- mesas Internacionales 2010, Tegucigalpa, Instituto Nacional de Estadística.

- INM y otros (2011). Encuesta sobre Migración en la Frontera Sur de México (antes EMIF GUAMEX), serie histórica 2004-2008, México, Instituto Nacional de Migración.

- Maguid, Mirta Alicia (2009). El estudio de la emigración internacional mediante los censos realizados en los países de origen: evaluación de resultados y recomendaciones, Notas de Población no 88, Año XXXVI, Santiago, CEPAL.

- OEA (2011). Migración Internacional en las Américas, Primer Informe del Sistema Continuo de Reportes de Migración Internacional en las Américas (SICREMI 2011), Washington.

- OIM (2011). Informe sobre las Migraciones en el Mundo 2011: Comunicar eficazmente sobre las Migraciones, Ginebra, Organización Internacional para las Migraciones.

- PEW Hispanic Center (2011). Hispanics of Honduran Origin in the United States, 2009. Washington, PEW. 